\title{
The Quest for Mindful Sleep: a Critical Synthesis of the Impact of Mindfulness-Based Interventions for Insomnia
}

\author{
Sheila N. Garland ${ }^{1,2} \cdot$ Eric S. Zhou ${ }^{3,4} \cdot$ Brian D. Gonzalez $^{5} \cdot$ Nicole Rodriguez $^{1}$
}

Published online: 5 July 2016

(C) Springer International Publishing AG 2016

\begin{abstract}
Mindfulness-based interventions (MBIs) for insomnia and sleep disturbances are receiving increasing clinical and research attention. This paper provides a critical appraisal of this growing area investigating the application of MBIs for people with insomnia and sleep disturbance. First, we discuss the theoretical justification for how mindfulness meditation practice may affect sleep processes. Second, we provide a focused review of literature published between January 1, 2012 and April 1, 2016 examining the impact of MBIs on sleep, broken down by whether insomnia or sleep disturbance was a primary or secondary outcome. Recommendations for future research are discussed.
\end{abstract}

Keywords Mindfulness $\cdot$ Meditation $\cdot$ Insomnia $\cdot$ Sleep disturbance $\cdot$ Intervention

This article is part of the Topical Collection on Behavioral Therapy

Sheila N. Garland

sheila.garland@mun.ca

1 Department of Psychology, Faculty of Science, Memorial University, 232 Elizabeth Avenue, St. John's, NF A1B 3X9, Canada

2 Division of Oncology, Faculty of Medicine, Memorial University, 300 Prince Phillip Dr., St. John's, NF A1B 3V6, Canada

3 Department of Pediatrics, Harvard Medical School, 25 Shattuck Street, Boston, MA 02115, USA

4 Dana-Farber Cancer Institute, 450 Brookline Avenue, Boston, MA 02215, USA

5 Rutgers Cancer Institute of New Jersey, 195 Little Albany Street, New Brunswick, NJ 08903, USA

\section{Introduction/Background}

Insomnia is a universal concern affecting individuals across the lifespan. A multi-national cross sectional survey of 25,579 individuals suggests that $35 \%$ of the population reports some difficulty initiating and maintaining sleep, or non-restorative sleep at least 3 days per week, with $10 \%$ reporting significant daytime consequences of poor sleep [1]. The most common treatment for trouble sleeping continues to be prescription sleep aids with recent estimates suggesting that $4 \%$ of the US population (approximately 11 million people) endorsed its use within the preceding 30 days [2]. However, sleeping aids are associated with a number of negative health outcomes including increased risk for motor vehicle accidents [3], falls and fractures in the elderly [4], the development of comorbid psychiatric [5] and medical conditions [6], and increased overall mortality [7], and may not be desired by patients with chronic medical illnesses [8]. Considering the possible risks associated with prescription sleep aid use and the lack of efficacy data in various populations with chronic illness $[9,10]$, it is important to provide patients with evidence-based alternatives that fit their unique needs.

Cognitive behavior therapy for insomnia (CBT-I) is a highly effective non-pharmacological intervention for insomnia that is considered first-line treatment for chronic insomnia disorder [11, 12]. Strong evidence demonstrates that CBT-I and hypnotic medications are equally effective in the shortterm, whereas the gains from CBT-I are significantly better maintained over time relative to hypnotic therapies [13-15]. Despite the endorsement of CBT-I as a first-line treatment for insomnia disorder from both the National Institutes of Health [11] and the American Academy of Sleep Medicine [12], several barriers to accessing CBT-I remain. First, there is a lack of awareness among the general public [16] and primary care providers [17] to the existence of, and evidence for, CBT-I. 
Second, despite solid efforts to increase the number of trained professionals [18] and provide alternative delivery models [19-21], the reach of CBT-I remains limited in large part to major cities and academic medical centers [22]. Lastly, insurance reimbursement for CBT-I can be poor, particularly if delivered by non-physician mental health providers (e.g., psychologists) [23].

Despite the evidence that CBT-I is an effective intervention with lasting effects [13-15], there are still a significant number of individuals whose insomnia does not fully respond to CBTI. In a study evaluating CBT-I for persistent insomnia in adults, $40 \%$ did not report a significant treatment response and $61 \%$ continued to experience insomnia symptoms following therapy [13]. One potential explanation is poor adherence to treatment [24]. Several core components of CBT-I (e.g., sleep restriction and stimulus control) can be difficult to implement and often result in a short-term worsening of symptoms and patient discomfort $[25,26]$. It is clear that though CBT-I must be considered a key component in the treatment of insomnia, it may not be the right approach for every individual.

The application of mindfulness-based interventions (MBIs) for insomnia and sleep disturbances is receiving increasing amounts of clinical and research attention. Mindfulness intentionally brings awareness to present moment thoughts or sensations with an attitude of acceptance, patience, openness, curiosity, and kindness which is formally practiced through meditation focused on purposefully directing ones attention towards the breath, body sensations, feelings, or thoughts [27]. The practice of mindfulness meditation has been incorporated into clinical interventions as a potential pathway to more effectively decrease perceived stress and emotional reactivity associated with a broad range of clinical and non-clinical conditions. Mindfulness-based techniques have recently been integrated into the treatment of insomnia because (1) they aim to directly reduce pre-sleep arousal, (2) their rationale is easily understandable, (3) they are attractive to many patients, and (4) they may avoid the short-term adverse consequences sometimes seen in CBT-I. Thus, use of MBIs for insomnia and sleep disturbance may serve as a potential alternative and/or supplement to CBT-I. This paper provides a critical appraisal of this growing area investigating the application of MBIs for people with insomnia and sleep disturbance. First, we will discuss the theoretical justification for how mindfulness meditation practice may exert its effects on sleep processes. Second, we will review the literature with a focus on recent research published between January 1, 2012 and April 1, 2016. This review will be broken down by whether insomnia or sleep disturbance was a primary or secondary outcome. Lastly, recommendations for future research will be provided.

\section{How Mindfulness Meditation May Impact Sleep}

The exact mechanism(s) by which mindfulness practice works to influence sleep processes has not yet been established. Lundh and Broman suggested mindfulness might be useful in the treatment of insomnia as a means to reduce pre-sleep cognitive and physiological arousal [28]. They suggested that people tend to engage in goal-directed and controlled information processing (e.g., problem solving and decision-making) during the day and that sleep is facilitated by cognitive deactivation and a reduction in the amount of controlled and strategic information processing. This cognitive deactivation is paralleled by physiologic deactivation characterized by a decrease in muscle tone and a slowing of the cardiovascular and respiratory systems. In this way, mindfulness may help facilitate cognitive deactivation and physiological de-arousal by allowing the individual to disengage from their daily concerns and strivings. The mindfulness principles of letting go, acceptance, and non-striving are theoretically congruent to the passive nature of sleep and encourages a shifting of one's relationship to sleep-related thoughts.

Etiological models of chronic insomnia have generally identified elevated cognitive and physiological arousal to be a key factor in the development and maintenance of sleep disturbance [29]. It is unclear whether the effects of mindfulness-based stress reduction (MBSR) are stronger for physiological or cognitive arousal reduction or whether these mechanisms are addressed simultaneously. Ong and colleagues have proposed a meta-cognitive model of mindfulness exerting its influence on two levels of sleep-related arousal, suggesting that primary arousal is directly related to the belief in ones inability to sleep and the consequences of poor sleep while secondary arousal is the emotional tone and degree of attachment related to the primary arousal [30]. This model hypothesizes that mindfulness might serve as a means of reinterpreting sleeplessness or reducing secondary arousal via detachment from particular modes of thinking.

Of all the repetitive thought patterns, the tendency to ruminate has been identified as an important contributing factor to the development and maintenance of insomnia [31]. Compared to good sleepers, people who engage in ruminative thinking have significantly lower sleep efficiency, worse sleep quality, spend more time awake after sleep onset, and their ruminative thoughts focus on daytime symptoms of fatigue, poor concentration, and low mood [32]. Mindfulness meditation may allow participants to disengage from ruminative processing. Increasing the objectivity through which internal experience is viewed is intended to change one's relationship to the thoughts, as opposed to changing the thoughts themselves and has been referred to as re-perceiving [33]. Shapiro, Carlson, Astin, and Freedman suggest that re-perceiving may account for the salutary effects of MBSR by increasing self-regulation, clarifying values, promoting cognitive, 
emotional and behavioral flexibility, and allowing for the development of tolerance for, or a desensitization to, difficult emotional states through exposure [33]. The hypothesized mechanisms of mindfulness were reviewed in a recent metaanalysis of 20 studies using mediation analyses of MBSR and mindfulness-based cognitive therapy (MBCT) [34]. The authors found consistent evidence for a reduction in repetitive negative thinking as a mechanism of the effect of mindfulness on psychological outcomes. As such, mindfulness-based programs that reduce rumination via meta-cognitive processes may provide a unique treatment option for insomnia [35]; however, further research is needed to properly investigate this possibility.

Others have postulated that mindfulness may provide a vehicle to counter the attentional biases that occur in patients with insomnia [36]. Attention bias in insomnia refers to the tendency to selectively pay attention to perceived internal or external threats to sleep or the consequences of poor sleep [37]. Mindfulness begins by training oneself to pay attention to present moment experience and to redirect attention back to the focal point (e.g., breathing) should it become distracted by some thought. With repetition, it becomes easier to recognize these biases and the practice of distancing oneself from these thoughts is hypothesized to result in reduced sleep-interfering emotional and cognitive arousal [36].

It is likely that the hypotheses presented above for how MBIs might impact sleep are interrelated and simultaneously explain different, but connected, pieces of the puzzle. Physiological arousal will be noticed and create cognitive arousal. Thinking patterns such as rumination or attentional bias create unpleasant emotional states, which produces an anticipation of harm, and results in increased physiological arousal. Because patients with insomnia vary in cognitive styles and arousal thresholds, MBI aims to reduce insomnia by addressing both cognitive and physiological arousal.

\section{The Evidence for Mindfulness-Based Interventions for Insomnia}

While this review is not intended to be a formal systematic review, we performed a thorough literature search of published research from January 1, 2012 to April 1, 2016 using PubMed, PsycInfo, and Medline and the following search terms: sleep, insomnia, mindfulness, mindfulness-based stress reduction, mindfulness-based cognitive therapy, MBSR, MBCT, mindfulness-based therapy for insomnia, and MBTI. Reference lists of the retrieved articles were also reviewed. We included articles that were published in English, conducted with adult populations, included a subjective or objective measure of sleep as a primary or secondary outcome, and used a mindfulness-based intervention. Eighteen studies were identified; 7 assessed sleep as a primary outcome, and 11 included sleep measures as a secondary outcome.

\section{Sleep as a Primary Target of Mindfulness-Based Interventions}

Of the seven studies that specifically investigated the impact of mindfulness-based interventions on sleep outcomes, six were randomized controlled trials. Five of the studies assessed the impact of MBIs in patients with clinically diagnosed insomnia while the remaining two assessed more general sleep disturbances. Three of the studies were conducted with adults (one study only included women), two with older adults, and two in patients with cancer. The total sample including all the studies was 407 with the samples of individual studies ranging from 12 to 111 . Of the six randomized controlled studies, five compared MBIs to different interventions and/or included active control groups. The details of these studies are provided in Table 1.

The type of MBIs investigated in these studies varied, and often a MBI was compared to another active nonmindfulness-based treatment. For example, Wong, Ree, and Lee [43] conducted a comparative effectiveness trial in 64 individuals with insomnia. All participants completed an individually delivered 4-week CBT-I intervention and then were randomly assigned to either four more sessions of cognitive therapy (CT) or mindfulness-based therapy (MBT) either immediately or after a 4-week waiting period. The MBT intervention focused on developing mindfulness skills through formal and informal meditation practice and participants were encouraged to practice before bed, upon awakening, and at any other time they desired during the day. There was a statistically and clinically significant effect of CBT-I at 4 weeks on insomnia severity, sleep continuity and quality, and psychological functioning. Treatment with an additional 4 weeks of CT or MBT was associated with further improvements, but no significant between-group differences were found. While the design is novel, the most significant limitation of this study is that the first author delivered all of the interventions. It is also not clear whether similar benefits would have been observed if participants had received an additional 4 weeks of CBT-I.

The effectiveness of a modified version of MBSR titled mindfulness-based cancer recovery (MBCR) compared to CBT-I was evaluated in a sample of 111 cancer survivors with insomnia [39]. Both interventions were delivered to groups over the course of 8 weeks. Using a non-inferiority margin of four points on the Insomnia Severity Index [45], MBCR demonstrated inferiority to CBT-I when assessed immediately after the program but was within the non-inferiority margin at the 3-month follow-up. CBT-I produced larger improvements in diary-measured sleep latency and sleep efficiency but both 


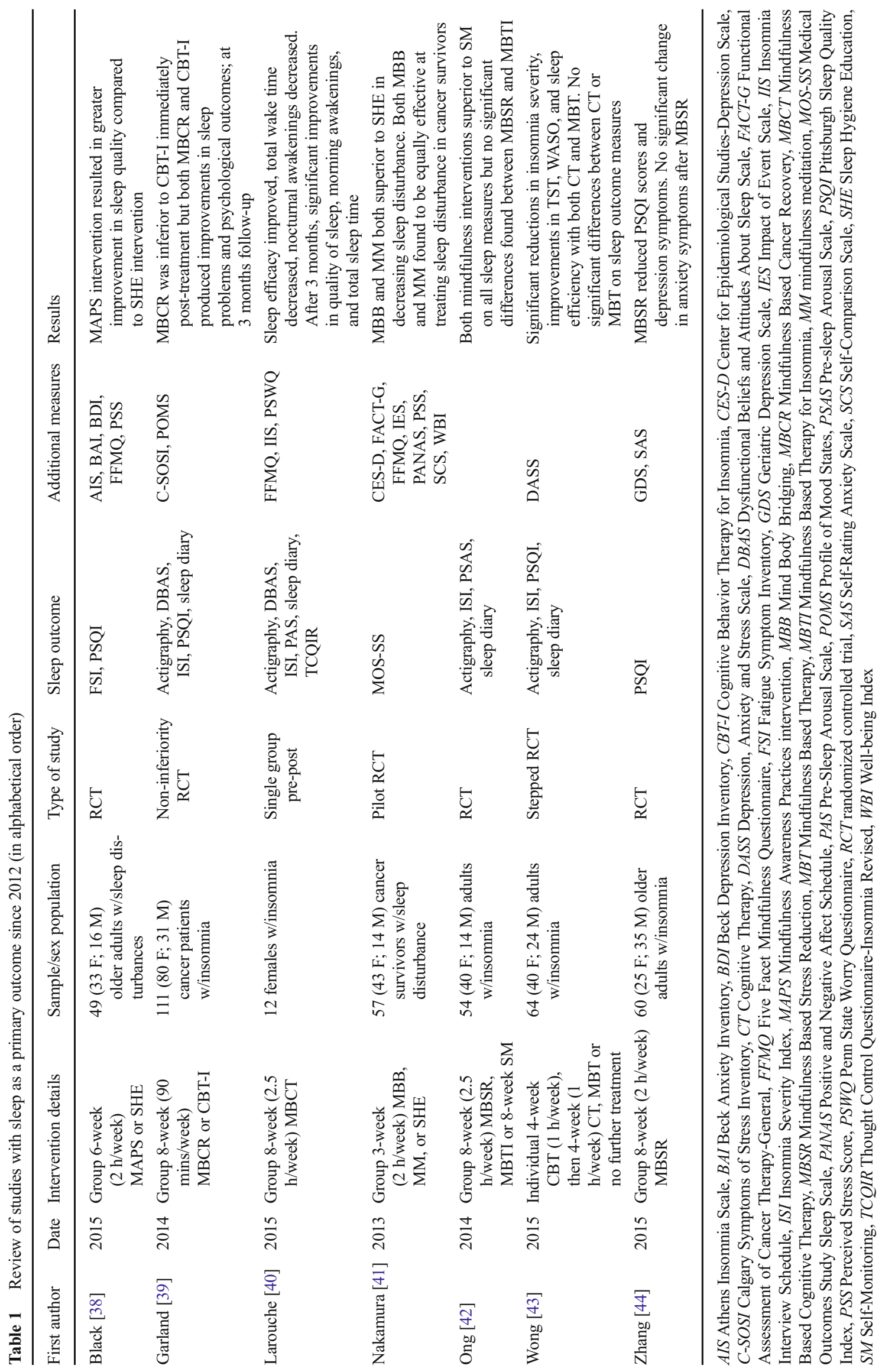


interventions significantly reduced amount of time spent awake after sleep onset and increased total sleep time. Significant reductions in symptoms of stress and psychological distress were also reported for both groups. The authors concluded that CBT-I remains the treatment of choice for insomnia but that MBIs should be considered a viable alternative for certain patients interested in an acceptance-based treatment approach. These may include patients interested in a mindfulness-based treatment approach, patients for whom CBT-I has been unsuccessful in the past, and patients unable or unwilling to implement challenging aspects of CBT-I such as sleep restriction (e.g., due to fatigue during chemotherapy for cancer). Future research is encouraged to apply an individualized or preference-based approach to evaluate the effectiveness of insomnia interventions.

Based on his meta-cognitive theory of insomnia, Ong et al. [42] developed an adaptation of MBSR specifically to insomnia called mindfulness-based therapy for insomnia (MBT-I). This 8-week group intervention teaches the behavioral strategies of CBT-I (sleep restriction, stimulus control, and sleep hygiene) and replaces the cognitive components of CBT-I with the discussion and practice of mindfulness meditation. The effectiveness of MBT-I was assessed by comparing it to an 8-week traditional MBSR group program and a selfmonitoring (SM) control group in a sample of 54 individuals with chronic insomnia disorder with follow-ups conducted at 3- and 6-months post-treatment. The effect sizes for reducing insomnia severity post-treatment using the Insomnia Severity Index (ISI; [46]) were 1.33, 2.07, and 0.01 for the MBSR, MBT-I, and SM groups, respectively. The 6-month effect sizes were 1.57 for MBSR and 2.56 for MBT-I. Response to treatment (defined as a $>7$ point reduction in ISI scores) and remission rates (defined as an ISI score $<8$ ) remained relatively constant in the MBSR group during the follow-up period (approximately $39 \%$ ). However, the MBT-I group continued to report gains after treatment with $50 \%$ achieving remission and $70 \%$ exhibiting a treatment response by 6 months. Because this study did not compare their intervention to standard CBT-I, it was not possible to determine the additive benefit of mindfulness training above the behavioral interventions provided in the MBT-I group.

Black et al. [38] extended their examination of MBI on sleep disturbances by incorporating a proposed biological marker of inflammation (nuclear factor-kB) that is associated with sleep disruption. The study compared a low-cost community MBI known as mindful-awareness practices (MAPs) to a sleep hygiene education (SHE) group in 49 older adults with sleep disturbances. The MAPs group was a weekly 2-h, six-session group-based course, designed to instruct participants in mindfulness meditation practice. The SHE intervention was matched for time, attention, expectancy, and group effect. The content of SHE consisted of psychoeducation about sleep and stress as well as relaxation training and sleep hygiene instructions. Only the MAPS group reported a significant improvement in sleep quality after program completion and improved sleep was associated with increased mindfulness. While no significant effect was observed for the secondary measure of nuclear factor-kB, this study will likely be followed by many more determined to discover whether improved sleep produced by non-pharmacological interventions can translate into improved health at a cellular level.

In sum, the evidence from randomized trials indicate that MBIs can contribute to reduced insomnia severity and sleep disturbance in healthy individuals, people with chronic disease, and older adults. These results do not appear to favor one particular type of meditation training program, any specific population, or age group. However, future research can compare the efficacy of various MBIs and identify groups of patients for whom MBIs are particularly effective.

\section{Sleep as a Secondary Target of Mindfulness-Based Interventions}

Of the 11 studies that investigated the impact of mindfulnessbased interventions on sleep as a secondary outcome, seven were randomized controlled trials. Five of the studies assessed the impact of MBIs on sleep in people with cancer, two studies were conducted in patients with depression, two studies were conducted in patients with pain conditions, one study was conducted in people with diabetes and coronary heart disease, and one in people reporting high levels of subjective stress. The sample size of individual studies ranged from 25 to 336 . Sleep was assessed using validated measures in 8 of the 11 studies, with the remaining three using single items from other measures. Details of these studies can be found in Table 2.

One novel study conducted by van der Zwan et al. [57] compared self-guided physical activity (PA), mindfulness meditation (MM), and heart rate variability biofeedback (BF) in a sample of 126 participants suffering from stress. All three interventions consisted of a single 2-h session where participants were instructed and practiced their assigned intervention: (1) the PA group was instructed to engage in vigorous exercise of their choosing; (2) the MM group was provided readings and a $\mathrm{CD}$ with guided meditations for home practice; (3) the BF group was provided with an infrared finger photoplethysmograph and a brochure with instructions on breathing exercises. Participants were instructed to do daily exercises at home increasing in duration from $10 \mathrm{~min}$ per day in the first week to $20 \mathrm{~min}$ per day at week 5 . A statistically significant improvement in sleep quality (measured by the Pittsburgh Sleep Quality Index; PSQI [58]) was demonstrated for the MM group only, though it did not translate into a clinically significant change. The primary intervention target (perceived stress) is a mechanism by which the mindfulness- 


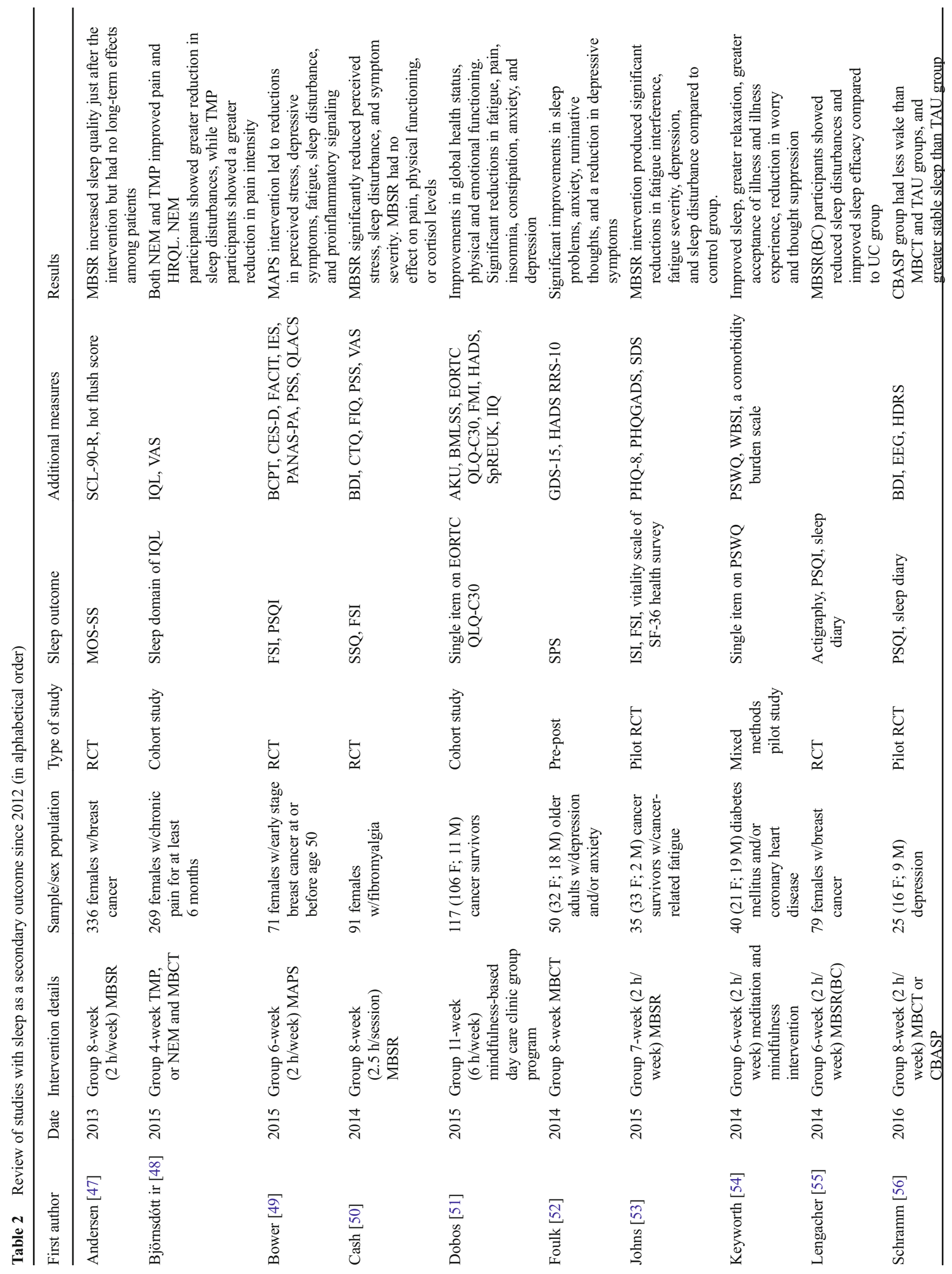




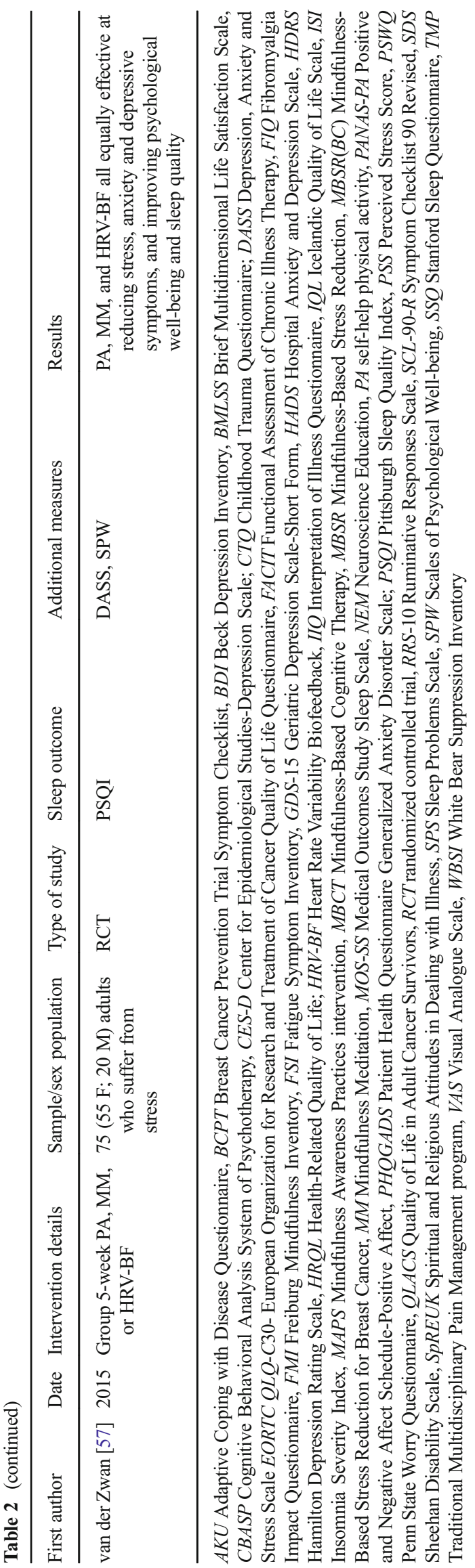

based practices are theorized to improve perceptions of overall sleep quality.

When considering the potential impact of MBSR on sleep outcomes, the work conducted by Lengacher et al. [55] provides an important cautionary lesson. The study authors included sleep as a secondary outcome in a randomized trial comparing a 6-week MBSR program in women with early stage breast cancer $(n=38)$ to a usual care control group $(n=41)$. The sample did not include individuals with clinically significant sleep issues at baseline. Specifically, the baseline total PSQI score was 8, and while this would not be characteristic of "good" sleepers [59], patients diagnosed with insomnia included in other intervention trials have typically reported PSQI values closer to $13[39,60]$. Not surprisingly, no subjective sleep improvements were noted, and the statistically significant increase in sleep efficiency, and decreased number of awakenings as measured by actigraphy had minimal clinical relevance. Additionally, the actigraph was only worn for 3 days at each assessment time, making it susceptible to one particularly good or poor night of sleep. In efforts to establish the efficacy of MBIs for insomnia and/or sleep disturbances, researchers must design trials with appropriate samples to avoid potential floor effects and allow the full impact of the intervention to be tested.

In all, the research investigating the impact of MBIs on sleep as a secondary outcome has considerable diversity. Of the remaining studies that assessed sleep as a secondary outcome of an MBI intervention, three found MBI significantly reduced subjective insomnia or sleep disturbance and six found no effect. However, these studies have several limitations and may have been underpowered to detect effects on insomnia or sleep disturbance. Specifically, a number of studies did not include validated stand-alone sleep measures; instead, sleep quality/insomnia was a single item part of a larger quality of life instrument. As noted above, the populations did not always present with significant sleep issues at baseline, making it a difficult test of MBIs ability to improve sleep outcomes. Finally, it is also not clear if the effects of MBI on sleep were direct or whether they were mediated by reductions in the primary symptom (e.g., pain, fatigue).

\section{Conclusion/Discussion}

The studies reviewed found significant impacts of MBIs on insomnia and sleep disturbance, and these effects were especially strong in studies primarily aimed at improving sleep. Findings were more mixed in studies that examined sleep as a secondary outcome, particularly those which did not seek to recruit a patient population who were experiencing active insomnia symptomatology. Although recent research has advanced the field of MBIs as a viable treatment option for 
patients with insomnia, critical questions remain about mechanism(s) of function and the delivery of treatment.

One important area for future examination is identifying the mechanisms by which MBIs improve insomnia symptoms. One theory posits that MBIs reduce insomnia through their impact on attention bias and improved emotion regulation; however, longitudinal meditational models are needed to test whether reductions in cognitive and physiological arousal from mindfulness training is associated with subsequent improvements in sleep. Further, the necessary MBI "dosage" is unclear. Future studies should compare several lengths and intensities of treatment to ensure efficacy is optimized without inconveniencing patients, as has been done with CBT-I [61]. This type of research would be helpful to develop a sense of treatment burden and will likely result in opportunities to refine MBIs in order to streamline the intervention process for patients.

As CBT-I is recognized to be front-line therapy, and hypnotic medications are commonly dispensed in the treatment of insomnia, it is necessary to directly compare the efficacy of MBIs to these interventions. To date, only one study has compared an MBI to the recommended first-line treatment, CBT-I [39], and one other has compared MBIs to hypnotic medications [62]. Both studies found no significant differences in long-term efficacy for reducing insomnia severity. In particular, one important benefit of CBT-I over pharmacologic therapy has largely been overlooked in the MBI literature. Studies have shown that whereas the efficacy of pharmacotherapy wanes after treatment is discontinued, CBT-I demonstrates efficacy for as long as 2 years after the intervention has completed [63]. Future studies of MBIs should include long-term follow-up periods now that evidence has conclusively shown the short- and medium-term efficacy for reducing insomnia $[38,42]$. Properly powered studies should aim to determine which of the various treatment options are most efficacious in both the short and long-term.

Beyond the direct comparison between MBIs and other treatment modalities, another opportunity for future study is determining which patients may benefit most from each type of therapy. Studies should compare the efficacy of MBIs in populations for which a component of one or more alternative treatment options may pose a significant barrier. For example, patients with some chronic illnesses (e.g., cancer, kidney disease) experience significant fatigue $[64,65]$ and may be unwilling or unable to adhere to the sleep restriction component of CBT-I. There may also be important patient factors that can moderate the impact of some treatment options, including severity of insomnia, age, and gender. Similarly, some patients may prefer a cognitive-behaviorally based treatment over one based in mindfulness theory. For example, Garland and colleagues reported drop-out rates of $52 \%$ and $15 \%$ within the first 3 weeks of starting MBSR and CBT-I groups, respectively, when participants were recruited for the trial without knowing what interventions were being compared [39]. Investigators should study whether patient preference may impact efficacy of, or retention in, MBIs for insomnia.

Future studies should also explore the benefits of administering MBIs in assorted modalities. Internet-delivered CBT-I has demonstrated efficacy comparable to that of face-to-face CBT-I $[20,66]$. Delivery via the Internet significantly increases the disseminability of an intervention, can reduce the cost considerably, and may eliminate other barriers to treatment for insomnia, including time constraints and difficulty traveling. We are unaware of any randomized clinical trials that have tested Internet-delivered MBIs for insomnia, but mobile and eHealth adaptations of MBIs in other populations have been developed and evaluated [67-69]. Future studies should capitalize on this and other opportunities to spread the reach of MBIs.

Acknowledgments Sheila N. Garland is funded in part by a PatientCentered Outcomes Research Institute (PCORI) Award (CER-140314292). Brian D. Gonzalez receives support from a NCI CRCHD research supplement grant R01CA185623-S1.

\section{Compliance with Ethical Standards}

Conflict of Interest Sheila N. Garland, Eric S. Zhou, Brian D. Gonzalez, and Nicole Rodriguez declare that they have no conflict of interest.

Human and Animal Rights and Informed Consent This article does not contain any studies with human or animal subjects performed by any of the authors.

\section{References}

1. Ohayon MM, Reynolds 3rd CF. Epidemiological and clinical relevance of insomnia diagnosis algorithms according to the DSM-IV and the International Classification of Sleep Disorders (ICSD). Sleep Med. 2009;10(9):952-60.

2. Chong Y, Fryer CD, Gu Q. Prescription sleep aid use among adults: United States, 2005-2010. NCHS Data Brief. 2013;127:1-8.

3. Hansen RN, Boudreau DM, Ebel BE, Grossman DC, Sullivan SD. Sedative hypnotic medication use and the risk of motor vehicle crash. Am J Public Health. 2015;105(8):e64-9.

4. Tannenbaum C, Diaby V, Singh D, Perreault S, Luc M, Vasiliadis HM. Sedative-hypnotic medicines and falls in community-dwelling older adults: a cost-effectiveness (decision-tree) analysis from a US Medicare perspective. Drugs Aging. 2015;32(4):305-14.

5. Chung KH, Li CY, Kuo SY, Sithole T, Liu WW, Chung MH. Risk of psychiatric disorders in patients with chronic insomnia and sedative-hypnotic prescription: a nationwide population-based follow-up study. J Clin Sleep Med. 2015;11(5):543-51.

6. Sivertsen B, Salo P, Pentti J, Kivimaki M, Vahtera J. Use of sleep medications and risk of cancer: a matched case-control study. Sleep Med. 2015;16(12):1552-5.

7. Kripke DF. Mortality risk of hypnotics: strengths and limits of evidence. Drug Saf. 2016;39(2):93-107. 
8. McHugh RK, Whitton SW, Peckham AD, Welge JA, Otto MW. Patient preference for psychological vs pharmacologic treatment of psychiatric disorders: a meta-analytic review. J Clin Psychiatry. 2013;74(6):595-602.

9. Riemann D, Spiegelhalder K, Espie C, Pollmacher T, Leger D, Bassetti C, et al. Chronic insomnia: clinical and research challenges - an agenda. Pharmacopsychiatry. 2011;44(1):1-14.

10. Rosenberg RP. Sleep maintenance insomnia: strengths and weaknesses of current pharmacologic therapies. Ann Clin Psychiatry. 2006;18(1):49-56.

11. National Institutes of Health. National Institutes of Health State of the Science Conference statement on manifestations and management of chronic insomnia in adults, June 13-15, 2005. Sleep. 2005;28(9):1049-57.

12. Schutte-Rodin S, Broch L, Buysse D, Dorsey C, Sateia M. Clinical guideline for the evaluation and management of chronic insomnia in adults. J Clin Sleep Med. 2008;4(5):487-504.

13. Morin CM, Vallieres A, Guay B, Ivers H, Savard J, Merette C, et al. Cognitive behavioral therapy, singly and combined with medication, for persistent insomnia: a randomized controlled trial. JAMA. 2009;301(19):2005-15.

14. Sivertsen B, Omvik S, Pallesen S, Bjorvatn B, Havik OE, Kvale G, et al. Cognitive behavioral therapy vs zopiclone for treatment of chronic primary insomnia in older adults: a randomized controlled trial. JAMA. 2006;295(24):2851-8.

15. Jacobs GD, Pace-Schott EF, Stickgold R, Otto MW. Cognitive behavior therapy and pharmacotherapy for insomnia: a randomized controlled trial and direct comparison. Arch Intern Med. 2004;164(17):1888-96.

16. Cheung JM, Bartlett DJ, Armour CL, Glozier N, Saini B. Insomnia patients' help-seeking experiences. Behav Sleep Med. 2014;12(2): 106-22.

17. Cheung JM, Atternas K, Melchior M, Marshall NS, Fois RA, Saini B. Primary health care practitioner perspectives on the management of insomnia: a pilot study. Aust J Prim Health. 2014;20(1):103-12.

18. Manber R, Carney C, Edinger J, Epstein D, Friedman L, Haynes PL, et al. Dissemination of CBTI to the non-sleep specialist: protocol development and training issues. J Clin Sleep Med. 2012;8(2): 209-18.

19. Ho FY, Chung KF, Yeung WF, Ng TH, Cheng SK. Weekly brief phone support in self-help cognitive behavioral therapy for insomnia disorder: relevance to adherence and efficacy. Behav Res Ther. 2014;63C:147-56.

20. Ritterband LM, Bailey ET, Thorndike FP, Lord HR, FarrellCarnahan L, Baum LD. Initial evaluation of an Internet intervention to improve the sleep of cancer survivors with insomnia. Psychooncology. 2011;21(7):695-705.

21. Vincent N, Lewycky S. Logging on for better sleep: RCT of the effectiveness of online treatment for insomnia. Sleep. 2009;32(6): $807-15$.

22. Perlis ML, Smith MT. How can we make CBT-I and other BSM services widely available? J Clin Sleep Med. 2008;4(1):11-3.

23. Fields BG, Schutte-Rodin S, Perlis ML, Myers M. Master's-level practitioners as cognitive behavioral therapy for insomnia providers: an underutilized resource. J Clin Sleep Med. 2013;9(10): 1093-6.

24. Stevens J. Behavioral economics strategies for promoting adherence to sleep interventions. Sleep Med Rev. 2015;23:20-7.

25. Riedel BW, Lichstein KL. Strategies for evaluating adherence to sleep restriction treatment for insomnia. Behav Res Ther. 2001;39(2):201-12.

26. Vincent NK, Hameed H. Relation between adherence and outcome in the group treatment of insomnia. Behav Sleep Med. 2003;1(3): 125-39.
27. Carlson LE. Mindfulness-based interventions for physical conditions: a narrative review evaluating levels of evidence. ISRN Psychiatry. 2012;2012:651583.

28. Lundh L, Broman JE. Insomnia as an interaction between sleepinterfering and sleep-interpreting processes. J Psychosom Res. 2000;49(5):299-310.

29. Bonnet MH, Arand DL. Hyperarousal and insomnia: state of the science. Sleep Med Rev. 2010;14(1):9-15.

30. Ong JC, Ulmer CS, Manber R. Improving sleep with mindfulness and acceptance: a metacognitive model of insomnia. Behav Res Ther. 2012;50(11):651-60.

31. Carney CE, Edinger JD, Meyer B, Lindman L, Istre T. Symptomfocused rumination and sleep disturbance. Behav Sleep Med. 2006;4(4):228-41.

32. Zoccola PM, Dickerson SS, Lam S. Rumination predicts longer sleep onset latency after an acute psychosocial stressor. Psychosom Med. 2009;71(7):771-5.

33. Shapiro SL, Carlson LE, Astin JA, Freedman B. Mechanisms of mindfulness. J Clin Psychol. 2006;62(3):373-86.

34. Gu J, Strauss C, Bond R, Cavanagh K. How do mindfulness-based cognitive therapy and mindfulness-based stress reduction improve mental health and wellbeing? A systematic review and metaanalysis of mediation studies. Clin Psychol Rev. 2015;37:1-12.

35. Carney CE, Harris AL, Moss TG, Edinger JD. Distinguishing rumination from worry in clinical insomnia. Behav Res Ther. 2010;48(6):540-6.

36. Larouche M, Cote G, Belisle D, Lorrain D. Kind attention and nonjudgment in mindfulness-based cognitive therapy applied to the treatment of insomnia: state of knowledge. Pathol Biol (Paris). 2014;62(5):284-91.

37. Harris K, Spiegelhalder K, Espie CA, MacMahon KM, Woods HC, Kyle SD. Sleep-related attentional bias in insomnia: a state-of-thescience review. Clin Psychol Rev. 2015;42:16-27.

38. Black DS, O'Reilly GA, Olmstead R, Breen EC, Irwin MR. Mindfulness meditation and improvement in sleep quality and daytime impairment among older adults with sleep disturbances: a randomized clinical trial. JAMA Intern Med. 2015;175(4):494 501.

39. Garland SN, Carlson LE, Stephens AJ, Antle MC, Samuels C, Campbell TS. Mindfulness-based stress reduction compared with cognitive behavioral therapy for the treatment of insomnia comorbid with cancer: a randomized, partially blinded, noninferiority trial. J Clin Oncol. 2014;32(5):449-57.

40. Larouche M, Lorrain D, Cote G, Belisle D. Evaluation of the effectiveness of mindfulness-based cognitive therapy to treat chronic insomnia. Eur Rev Appl Psychol. 2015;65:115-23.

41. Nakamura Y, Lipschitz DL, Kuhn R, Kinney AY, Donaldson GW. Investigating efficacy of two brief mind-body intervention programs for managing sleep disturbance in cancer survivors: a pilot randomized controlled trial. J Cancer Surviv. 2013;7(2):165-82.

42. Ong JC, Manber R, Segal Z, Xia Y, Shapiro S, Wyatt JK. A randomized controlled trial of mindfulness meditation for chronic insomnia. Sleep. 2014;37(9):1553-63.

43. Wong MY, Ree MJ, Lee CW. Enhancing CBT for chronic insomnia: a randomised clinical trial of additive components of mindfulness or cognitive therapy. Clin Psychol Psychother. 2015. doi:10.1002/cpp.1980.

44. Zhang JX, Liu XH, Xie XH, Zhao D, Shan MS, Zhang XL, et al. Mindfulness-based stress reduction for chronic insomnia in adults older than 75 years: a randomized, controlled, single-blind clinical trial. Explore (NY). 2015;11(3):180-5.

45. Savard MH, Savard J, Simard S, Ivers H. Empirical validation of the Insomnia Severity Index in cancer patients. Psychooncology. 2005;14(6):429-41. 
46. Bastien $\mathrm{CH}$, Vallieres A, Morin CM. Validation of the Insomnia Severity Index as an outcome measure for insomnia research. Sleep Med. 2001;2(4):297-307.

47. Andersen SR, Wurtzen H, Steding-Jessen M, Christensen J, Andersen KK, Flyger H, et al. Effect of mindfulness-based stress reduction on sleep quality: results of a randomized trial among Danish breast cancer patients. Acta Oncol. 2013;52(2):336-44.

48. Bjornsdottir SV, Arnljotsdottir M, Tomasson G, Triebel J, Valdimarsdottir UA. Health-related quality of life improvements among women with chronic pain: comparison of two multidisciplinary interventions. Disabil Rehabil. 2016;38(9):828-36.

49. Bower JE, Crosswell AD, Stanton AL, Crespi CM, Winston D, Arevalo J, et al. Mindfulness meditation for younger breast cancer survivors: a randomized controlled trial. Cancer. 2015;121(8): 1231-40.

50. Cash E, Salmon P, Weissbecker I, Rebholz WN, Bayley-Veloso R, Zimmaro LA, et al. Mindfulness meditation alleviates fibromyalgia symptoms in women: results of a randomized clinical trial. Ann Behav Med. 2015;49(3):319-30.

51. Dobos G, Overhamm T, Bussing A, Ostermann T, Langhorst J, Kummel $\mathrm{S}$, et al. Integrating mindfulness in supportive cancer care: a cohort study on a mindfulness-based day care clinic for cancer survivors. Support Care Cancer. 2015;23:2945.

52. Foulk MA, Ingersoll-Dayton B, Kavanagh J, Robinson E, Kales HC. Mindfulness-based cognitive therapy with older adults: an exploratory study. J Gerontol Soc Work. 2014;57(5):498-520.

53. Johns SA, Brown LF, Beck-Coon K, Monahan PO, Tong Y, Kroenke K. Randomized controlled pilot study of mindfulnessbased stress reduction for persistently fatigued cancer survivors. Psychooncology. 2015;24(8):885-93.

54. Keyworth C, Knopp J, Roughley K, Dickens C, Bold S, Coventry P. A mixed-methods pilot study of the acceptability and effectiveness of a brief meditation and mindfulness intervention for people with diabetes and coronary heart disease. Behav Med. 2014;40(2):53-64.

55. Lengacher CA, Reich RR, Paterson CL, Jim HS, Ramesar S, Alinat $\mathrm{CB}$, et al. The effects of mindfulness-based stress reduction on objective and subjective sleep parameters in women with breast cancer: a randomized controlled trial. Psychooncology. 2014;24(4):424-32.

56. Schramm PJ, Zobel I, Monch K, Schramm E, Michalak J. Sleep quality changes in chronically depressed patients treated with mindfulness-based cognitive therapy or the cognitive behavioral analysis system of psychotherapy: a pilot study. Sleep Med. 2016;17:57-63.

57. van der Zwan JE, de Vente W, Huizink AC, Bogels SM, de Bruin EI. Physical activity, mindfulness meditation, or heart rate variability biofeedback for stress reduction: a randomized controlled trial. Appl Psychophysiol Biofeedback. 2015;40(4):257-68.

58. Buysse DJ, Reynolds 3rd CF, Monk TH, Berman SR, Kupfer DJ. The Pittsburgh Sleep Quality Index: a new instrument for psychiatric practice and research. Psychiatry Res. 1989;28(2):193-213.

59. Beck SL, Schwartz AL, Towsley G, Dudley W, Barsevick A. Psychometric evaluation of the Pittsburgh Sleep Quality Index in cancer patients. J Pain Symptom Manage. 2004;27(2):140-8.

60. Espie CA, Fleming L, Cassidy J, Samuel L, Taylor LM, White CA, et al. Randomized controlled clinical effectiveness trial of cognitive behavior therapy compared with treatment as usual for persistent insomnia in patients with cancer. J Clin Oncol. 2008;26(28):4651-8.

61. Edinger JD, Wohlgemuth WK, Radtke RA, Coffman CJ, Carney CE. Dose-response effects of cognitive-behavioral insomnia therapy: a randomized clinical trial. Sleep. 2007;30(2):203-12.

62. Gross CR, Kreitzer MJ, Reilly-Spong M, Wall M, Winbush NY, Patterson R, et al. Mindfulness-based stress reduction versus pharmacotherapy for chronic primary insomnia: a randomized controlled clinical trial. Explore (NY). 2011;7(2):76-87.

63. Morin CM, Colecchi C, Stone J, Sood R, Brink D. Behavioral and pharmacological therapies for late-life insomnia: a randomized controlled trial. JAMA. 1999;281(11):991-9.

64. Jim HS, Evans B, Jeong JM, Gonzalez BD, Johnston L, Nelson AM, et al. Sleep disruption in hematopoietic cell transplantation recipients: prevalence, severity, and clinical management. Biol Blood Marrow Transplant. 2014;20(10):1465-84.

65. Evans BJ, Phillips KM, Gonzalez BD, Apte S, Small BJ, Jacobsen $\mathrm{PB}$, et al. Psychosocial resources and sleep disturbance before chemotherapy for gynecologic cancer. J Psychosoc Oncol. 2016;34(12):60-76.

66. Riemann D, Perlis ML. The treatments of chronic insomnia: a review of benzodiazepine receptor agonists and psychological and behavioral therapies. Sleep Med Rev. 2009;13(3):205-14.

67. Younge JO, Wery MF, Gotink RA, Utens EM, Michels M, Rizopoulos D, et al. Web-based mindfulness intervention in heart disease: a randomized controlled trial. PLoS One. 2015;10(12): e0143843.

68. Zernicke KA, Campbell TS, Speca M, McCabe-Ruff K, Flowers S, Carlson LE. A randomized wait-list controlled trial of feasibility and efficacy of an online mindfulness-based cancer recovery program: the eTherapy for cancer applying mindfulness trial. Psychosom Med. 2014;76(4):257-67.

69. Plaza I, Demarzo MM, Herrera-Mercadal P, Garcia-Campayo J. Mindfulness-based mobile applications: literature review and analysis of current features. JMIR Mhealth Uhealth. 2013;1(2):e24. 